\title{
An Optimized Resonant-cap Ka-band IMPATT Oscillator for Broadband Communication
}

\author{
L.P. Mishra ${ }^{1}$, M.N. Mohanty $\star^{2}$, S. Chakraborty ${ }^{3}$, M. Mitra ${ }^{4}$ \\ ${ }^{1,2}$ Dept. of ECE, SOA University, Bhubaneswar, India \\ ${ }^{3}$ Dept. of ECE, MCKV Institute of Engineering, Howrah, India \\ ${ }^{4}$ Dept. of ENTC, IIEST, Shibpur, West Bengal, India \\ ${ }^{*}$ Corresponding author, e-mail: mihirmohanty@soauniversity.ac.in
}

\begin{abstract}
Demand for capacity and bandwidth increasing day-by-day in the area of wireless communication. One of the solutions is the utilization of frequency bands for broadband communication that is related to satellite communication. Mostly Ka-band is used in satellite communication. In this paper, an attempt is taken to design an optimized resonant-cap Ka-band oscillator. To obtain oscillation from Kaband IMPATT device, it has been embedded in a resonant cavity for which resonant cap structure is used. From this study, it is found that the supply D.C voltage from the top of the diode provides a matching network between the diode and the load to achieve maximum power. Keeping the breakdown voltage of the IMPATT diode fixed, the variation of bias current helps for detailed study and mechanically tuning has been done with a variation of cap height, Cap diameter as well as by varying the position of the short using sliding short tuner and verified experimentally. Optimization result is made to get maximum output with maximum efficiency for an optimized cap height, diameter, and position of sliding short tuner. With these facts the work is presented difference tuning methods for the resonant cap IMPATT oscillator.
\end{abstract}

Keywords: resonant cap IMPATT oscillator, Ka-band IMPATT oscillator, mechanical tuning of IMPATT, sliding short tuner

Copyright $(2015$ Institute of Advanced Engineering and Science. All rights reserved.

\section{Introduction}

Wireless and satellite communications are rapidly growing industries which are slated for explosive growth in emerging countries as well as countries with advanced economies. From the radio to satellite TV, the Internet to cellular communication, people are communicating more now than ever before. And it is precisely this communication that is causing exponential growth in science and technology standard of living and economic power. This fact alone is what separates the first world from the developing. The research in the area of wireless communication has been progress in many ways. Authors have been working on MIMO techniques for efficient communication. Similarly the mobile equipment location and velocity have been analyzed for the same. Nevertheless the handoff has been left for efficient and seamless service by many researchers [1-4]. But the dominant trend in wireless communication systems is towards broadband applications such as multimedia file transfer, video transmission and Internet access. These applications require much higher data transmission rates than those currently used for voice transmission applications. To achieve these higher data rates, substantially larger bandwidths and higher carrier frequencies are required. A key roadblock to implementing these systems at K-band $(18-26.5 \mathrm{GHz})$ and $\mathrm{Ka}$-band $(26.5-40 \mathrm{GHz})$ is the need to develop hardware which meets the requirements for high data rate transmission in a cost effective manner.

IMPATT (IMPact Avalanche ionization Transit Time) diode is a PN Junction diode operates at reverse bias to break down and can generate RF power, when it is properly embedded in a resonant cavity. In course of research different structures of IMPATT diodes are being developed by different scientists using different semiconductor materials. These days IMPATT diode can operate up to a frequency of $450 \mathrm{GHZ}$. Thus, it is covering microwave, millimeter wave, sub-millimeter wave range of frequencies. Because of the availability of these HF ranges, IMPATT diode day-by-day becoming very important in the field of different type of 
communications mentioned above and also becoming a strategic device for the use of defense people.

By means of different methods of tuning IMPATT diodes can be turned over a wide frequency range. In our problem the IMPATT diode had the optimum frequency of $36 \mathrm{GHZ}$ (Kaband), but these diodes can give a reasonable amount of power from a frequency range of 34 to $37 \mathrm{GHZ}$ and can offer many sub-bands under Ka-band for different types of communication. Though here the authors have indigenously developed the resonant cavity with a post and cap structure, but the suitability of this method is compared at the end with other methods of tuning such as varying bias current, position of sliding short tuner etc.

The resonant cap IMPATT oscillator was first reported by Lee [5] for the millimeter wave band an owing to their simplicity of construction and good performance in microwave and millimeter wave frequency bands made it very attractive. A wide variation of mechanical tuning by varying the sliding short tuner for a resonant cap IMPATT was observed by Misawa and Kenyon [6] for low frequency IMPATT diodes. In 1972 Groves and Lewis [7] vary various structural parameters of the resonant cap to obtain maximum power output at low frequency. Mallik et.al [8] in 1983 derived an empirical formula using their experimental data related to cap diameter and the oscillation frequency. Computer aided design and analysis of broadband communication performed by authors [9-10]. Also, the IMPATT oscillator has been utilized for the same and used for Ka band [11-14]

\section{Design}

Demands on wireless communications have motivated the development of RF front-end circuits toward tens of gigahertz to achieve high-data-rate transmission. Ka band oscillators are especially designed for low cost commercial applications. The explosion of cellular phones and the Internet follows the next large communications technology, broadband communication research for large amount of bandwidth that translates to large amount of information.

In this piece of work, it has been tried for the design of Resonant cap IMPATT Oscillator for Ka Band operation. It can be useful in satellite broadband communication further it has been optimized using mechanical tuning. Authors have used a resonant cap cavity in which the device is placed between the bottom broad wall of a millimeter wave guide and the bottom face of a circular metal disc in the resonant cap, as shown in Figure 1. A constant current power supply is used as a bias through the post and the disc structure of the resonant cap. The purpose is to study the effect of the cap diameter and cap height of a resonant cap IMPATT oscillator and the effect of plane of the short on the oscillation frequency and power output. The output power can be optimized when the device-circuit interaction will take place. The basic theory of the design is explained as follows.

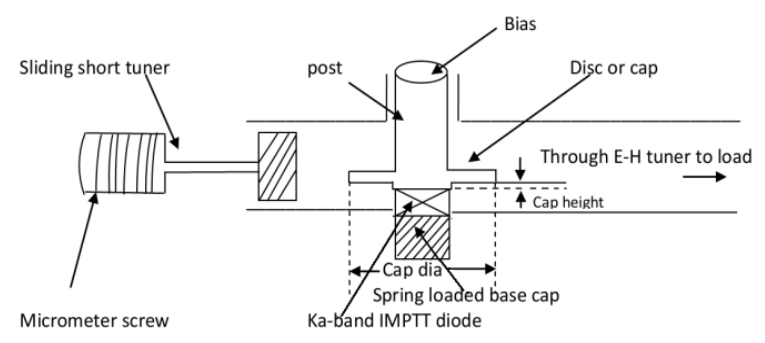

Figure 1. Schematic representation of Ka band IMPATT Oscillator

The resonant cap consists of disc-post structure with a flat disc in the $\mathrm{H}$ plane. The disc and bottom broad wall of the waveguide forms a radial line cavity around the diode. The generated microwave power propagates in the radial direction of the disc, and is getting coupled to the waveguide through the open vertical edges of the radial cavity. The disc of the resonant cap acts as an impedance transformer and helps to match the impedances between the low impedance diode and the high impedance waveguide so that maximum generated RF energy can be transferred to the load.

TELKOMNIKA Vol. 16, No. 3, December 2015: $553-558$ 
These post and disc structure also provides a low pass $\pi$ type filter to bypass the ripple present in the power supply. The resonant cavity is such that it can be also used as an external heat sink for the IMPATT diode.

When direct current (DC) bias is applied to the input terminals of the oscillator, AC energy is produced at the output terminals. AC signal and radio frequency (RF) are synonymous, in the simplest terms. Also an oscillator can be defined as a DC to RF energy converter.

The total current is composed of conduction current and displacement current only because the diffusion current is negligibly small. The total current density is given by the expression.

$$
I_{\text {total }}=q\left(n, v_{g n}+p, v_{g p}\right)+\frac{\delta(\epsilon E)}{\delta t}
$$

The spatial variation of electric field in the space charge layer is obtained from Poisson's equation as:

$$
\begin{aligned}
& \frac{\delta E}{\delta x}=\frac{q}{\epsilon}\left[\left(N_{D}-N_{A}\right)+(\mathrm{p}-\mathrm{n})\right] \\
& \text { Where: } \\
& \mathrm{E}=\text { electric field, } \\
& \mathrm{q}=\text { electronic charge, } \\
& \mathrm{N}_{\mathrm{D}}=\text { ionized donor density, } \\
& \mathrm{N}_{\mathrm{A}}=\text { ionized acceptor density, } \\
& \mathrm{p}=\text { hole density, } \\
& \mathrm{n}=\text { electron density, } \\
& \epsilon=\text { permittivity of the semiconductor. }
\end{aligned}
$$

The device negative resistance and reactance are obtained from the following:

$$
\begin{aligned}
& Z_{R}=\int_{0}^{W} X d x . \\
& \text { Conductance } G=\frac{Z_{R}}{Z_{R}^{2}+Z_{X}^{2}} \\
& \text { Susceptance } B=-\frac{Z_{x}}{Z_{R}^{2}+Z_{X}^{2}}
\end{aligned}
$$

With electromagnetic radiation effects much more subtle at Ka-band frequencies, and with greater susceptibility to parasitic effects and losses, significantly greater attention is given in the technique, particularly at high power levels. The experimental setup, and its result are explained in the following section 3 .

\section{Experimental Setup and Results}

This work is going to present different tuning methods for resonant cap IMPATT Oscillator. But the study of an amplitude control and stability is a different aspect and thus not considered here. The experimental arrangement for the study of resonant cap Ka-band IMPATT oscillator is as shown in Figure 2.

Indigenously developed Ka-band SDR IMPATT diode is used. The diode oscillates over a range of 34 to $38 \mathrm{GHz}$ with a breakdown voltage of $45 \mathrm{v}$. A maximum power output of $90 \mathrm{mw}$ for a maximum dc bias current of $140 \mathrm{~mA}$ for occurrence of the oscillation. A variation of oscillation frequency and power output due to the variation of dc bias current is shown in Figure 3 . In the graph the power output increases non-linearly with dc bias current and reaches a maximum value and then starts to fall gradually but the frequency increases more or less linearly. It is because in the G-B slot of Figure 4 where the circuit locus takes a turn. Therefore, the negative conductance corresponding to the point of intersection between the active and passive characteristics decreases after reaching a maximum. 


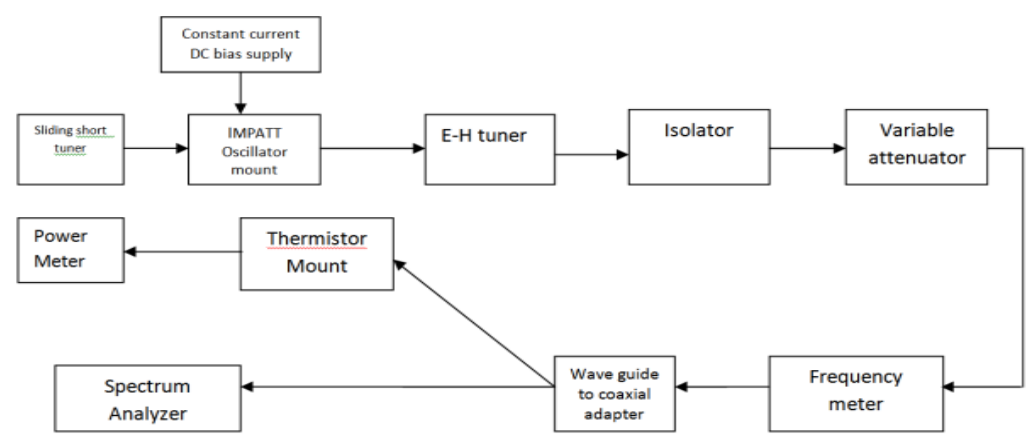

Figure 2. Experimental arrangement of resonant cap Ka-band IMPATT oscillator

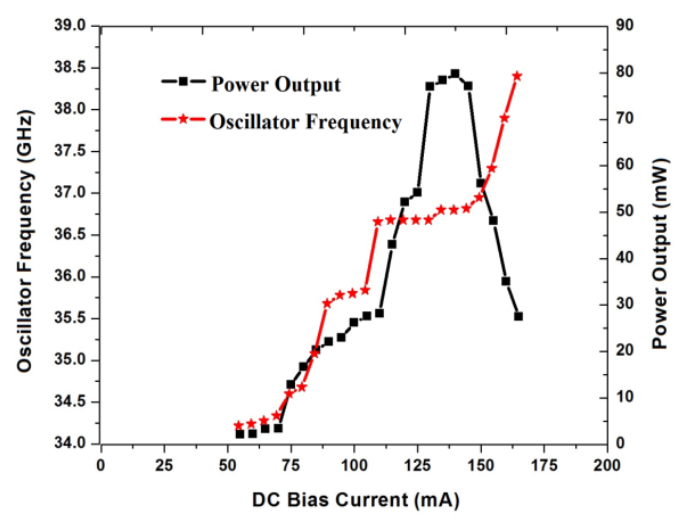

Figure 3. Plot of oscillation frequency and power output vs DC bias current

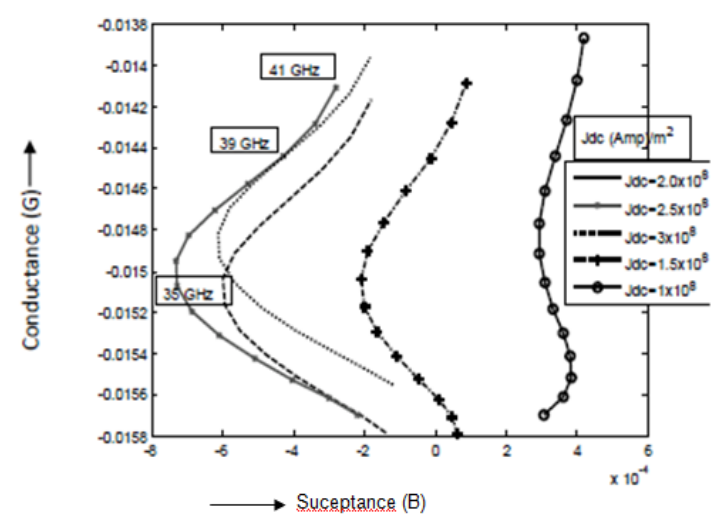

Figure 4. G-B plot of Si- IMPATT for different dc bias current density

The dimension of the resonant cap has a major impact on output power and frequency. As [8], the relation between frequency and cap dimension is depicted as:

$$
f=C / \lambda=\frac{0.45 C}{D+L}
$$

Where $D$ is the diameter of the resonant cap and $L$ is the height of the resonant cap. It has been experienced with the variation of the cap height, keeping the cap diameter constant and viceversa.

IMPATT diode always operates at reverse bias to break down. As the breakdown voltage is fixed, it shows with varying the bias current so that the tuning is done. This experimental result is shown in Table $1(\mathrm{a})$ and $1(\mathrm{~b})$ respectively.

Table 1(a). Variation of output power and frequency with cap height for a cap diameter of $(\mathrm{D})=2.7 \mathrm{~mm}$

\begin{tabular}{ccc}
\hline Cap Height $(\mathrm{mm})$ & Output Power $(\mathrm{mW})$ & Frequency $(\mathrm{GHz})$ \\
\hline 1 & 53.1 & 39.48 \\
1.1 & 60.24 & 35.52 \\
1.2 & 66.95 & 34.61 \\
1.3 & 73.9 & 33.75 \\
1.4 & 82.25 & 32.92 \\
1.5 & 85.01 & 32.14 \\
1.6 & 89.01 & 31.4 \\
1.7 & 87.25 & 30.68 \\
1.8 & 85.29 & 30 \\
1.9 & 79.79 & 29.34 \\
2 & 73.01 & 28.72 \\
\hline
\end{tabular}

TELKOMNIKA Vol. 16, No. 3, December 2015 : 553 - 558 
Table 1(b). Variation of output power and frequency with cap diameter for a cap height $=1 \mathrm{~mm}$

\begin{tabular}{ccc}
\hline Cap Diameter $(\mathrm{D})(\mathrm{mm})$ & Output Power $(\mathrm{mW})$ & Frequency $(\mathrm{GHz})$ \\
\hline 2.1 & 32.01 & 43.54 \\
2.3 & 42.34 & 40.91 \\
2.5 & 60.67 & 34.57 \\
2.7 & 74.85 & 36.49 \\
2.9 & 84.85 & 34.61 \\
3.1 & 87.41 & 32.93 \\
3.3 & 77.25 & 31.4 \\
3.5 & 59.15 & 30 \\
3.7 & 43.14 & 28.72 \\
3.9 & 32.22 & 27.55 \\
4.1 & 24.12 & 26.47 \\
\hline
\end{tabular}

\subsection{Mechanical Tuning}

As mentioned that the paper dealing with optimal tuning of IMPATT diode to get an idea about the best method for tunning so that a diode can operate over a wide range. Variation of oscillation frequency of the oscillator by varying mechanical parameters of the resonant cavity, such as cap height, cap diameter and the position of the sliding short tuner is called mechanical tuning. The dependence of the oscillation frequency on the cap height and cap diameter was investigated and presented the Table $1(\mathrm{a})$ and $(\mathrm{b})$. It is observed that the oscillation frequency decreases with the increase of cap diameter and cap height as per the relation. " $(D+L) / \lambda=K$ " given by Groves and Lewis and Mallik et.al where ' $K$ ' is a constant and equal to 0.45 and $\lambda$ is the operating wavelength ' $D$ ' is the cap diameter and ' $\lambda$ ' is the post length. The above formula tells that for a particular value of ' $D$ ' and ' $L$ ' the corresponding frequency when equal to the optimum design frequency of the diode then the power output is maximum.

In the first method of tuning a variation of bias current from a range of 50 to $160 \mathrm{~mA}$ results a variation in the frequency range of 34 to $38 \mathrm{GHz}$. Again by varying the cap height or cap diameter a frequency variation of $28 \mathrm{GHz}$ to $40 \mathrm{GHz}$ is possible. On the other hand a frequency variation of 34 to $37 \mathrm{GHz}$ is possible for a variation of the position of sliding short tuner 3 to $8 \mathrm{~mm}$ range. But from the diode a maximum power output of about $85 \mathrm{~mW}$ on an average is obtained in all the methods and at a distance of $\lambda / 2$ from the position of sliding short tuner.

The variation of power and frequency due to variable position of slide soft tuner is plotted in Figure 7. For a cavity resonator the frequency of oscillation is given by:

$$
f=C / 2 \sqrt{\left(\frac{m}{a}\right)^{2}+\left(\frac{n}{b}\right)^{2}+\left(\frac{p}{d}\right)^{2}}
$$

Where ' $a$ ' is the broad plane dimension, ' $b$ ' is the narrow plane dimension and ' $d$ ' is the length of the rectangular cavity resonator. Now variation of the position of sliding short tuner represents the variation of the length of the cavity and thus resonant frequency is varying. The graph of Figure 5 is showing the power at different resonant and anti resonant condition of the cavity.

The output spectrum of the resonant cap Ka-band IMPATT oscillator was investigated by using a spectrum analyzer and the resulting photograph is shown in Figure 6.

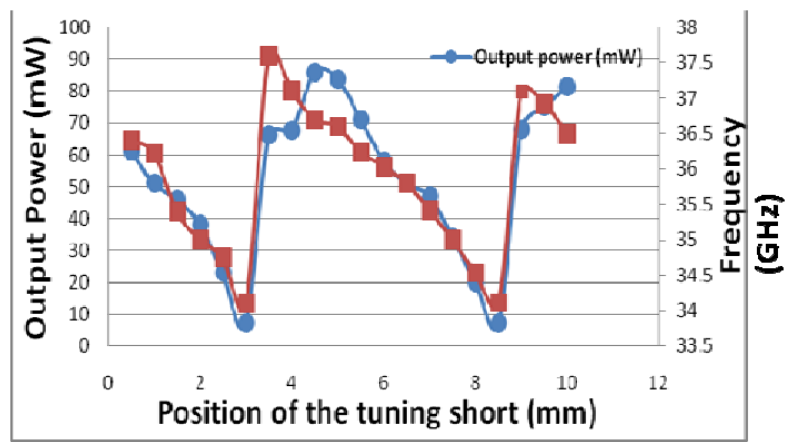

Figure 5. Variation of output power and frequency with position of the sliding short tuner 


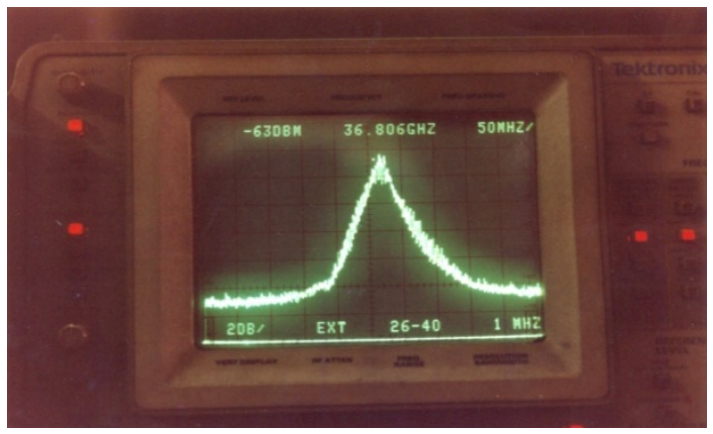

Figure 6. Output spectrum of resonant cap Ka-band IMPATT oscillator

\section{Conclusion}

Earlier works were related to tunning of IMPATT oscillator at X-band, but our paper is for Ka band Oscillation. In this paper three different methods of tuning of a Ka-band IMPATT oscillator are described. Out of these three methods variation of cap diameter or cap height gives a wide range for tuning of about $12 \mathrm{GHz}$ but in the other two methods, which are very easy but gives a frequency range of variation of only $3 \mathrm{GHz}$. Thus this paper gives a clear insight to the designer and user of Ka-band IMPATT oscillator. A coherent study of tuning properties of resonant cap IMPATT oscillator at Ka-band has been carried out by mechanical and electronic means. It is experimentally observed that output power of IMPATT oscillator passes through a maximum, with an optimum combination of cap diameter and cap height. Thus after comparison, it is being found that the method of tuning by variation of cap height and cap diameter shows the effective result.

\section{References}

[1] Mihir N Mohanty, Monalisa Bhol, Laxmi Prasad Mishra, Sanjat Kumar Mishra. Design and Analysis of Mimo System for UWB Communication. International Journal of Wireless \& Mobile Networks (IJWMN). 2014; 6(2): 101-109.

[2] Mihir Narayan Mohanty. Performance Analysis of MIMO Wirelss System with Array Antenna. ARPN Journal of Engineering and Applied Sciences. 2013; 8(10): 806-810.

[3] Mihir Narayan Mohanty, Laxmi Prasad Mishra, Saumendra Kumar Mohanty. Design of MIMO SpaceTime Code for High Data Rate Wireless Communication. IJCSE. 2011; 3(2): 693-697.

[4] S Bandopadhaya, LP Mishra, D Swain, Mihir N Mohanty. Design of DFE Based MIMO Communication System for Mobile Moving with High Velocity. International Journal of Computer Science and Information Technologies. 2014; 1(5): 319-323.

[5] CA Lee, L Baldorf, W Weighman, G Kaminsky. Technological development evolving from Research on Read Diode. IEEE Trans. On Electron Devices. 1966; 3: 75-180.

[6] T Miswa, ND Kenyon. An Oscillator Circuit with CAP structure for millimeter wave IMPATT diode. IEEE Trans. On Microwave Theory \& Tech. 1970; 18: 969.

[7] IS Groves, DE Levis. Resonant Cap structure for IMPATT diodes. Electron. Lett. (G.B). 1972; 8: 98.

[8] KK Mallik, M Sridharan, SK Roy. Some studies on the effect of Resonant-cap on the oscillator performance of x-band Gats and Si IMPATT oscillators. JIETE. 1983; 29: 215.

[9] Subal Kar, S Bhanja, S Sasmal. Computer-analytical Characterization of Resonant-cap Circuit for Microwave Oscillators and Power Combiners. Progress In Electromagnetics Research Symposium. Prague, Czech Republic. 2007: 27-30.

[10] Subal Kar. Computer-aided Design and Characterization of a Broad-band Millimeter-wave Source at 34GHz. Progress in Electromagnetics Research Symposium. Prague, Czech Republic. 2007: 27-30.

[11] Tapas Kumar Pal, JP Banerjee. Study of Efficiency of Ka-band IMPATT Diodes and Oscillators around Optimized condition. International Journal of Advanced Science and Technology. 2011; 26.

[12] Tapas Kumar Pal, JV Prasad, JP Banerjee. Study of Various Tuning properties and Injection Locking of Resonant-cap IMPATT Oscillator. International Journal of Engineering and Technology. 2010. 2(5): 329-335.

[13] Manidipa Nath. Design and Characterization of Microstrip IMPATT Oscillator. International Journal of Advanced Research in Computer and Communication Engineering. 2013; 2(11).

[14] Han-Young Lee, Wan-Sik Kim. The MMIC VCO Design for Wireless Systems at Ka-Band. Journal of Electrical Engineering \& Technology. 2010; 5(1): 151-155. 